\title{
Ability to predict repetitions to momentary failure is not perfectly accurate, though improves with resistance training experience
}

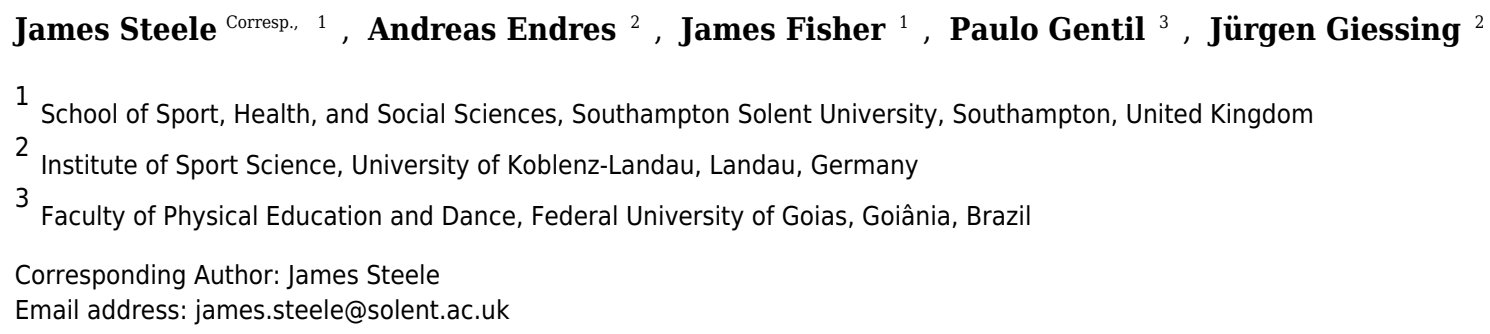

'Repetitions in Reserve' (RIR) scales in resistance training (RT) are used to control effort but assume people accurately predict performance a priori (i.e. the number of possible repetitions to momentary failure [MF]). This study examined the ability of trainees with different experience levels to predict number of repetitions to MF. One hundred and forty one participants underwent a full body RT session involving single sets to MF and were asked to predict the number of repetitions they could complete before reaching MF on each exercise. Participants under predicted the number of repetitions they could perform to MF (Standard error of measurements [95\% confidence intervals] for combined sample ranged between 2.64 [2.36 to 2.99] and 3.38 [3.02 to 3.83]). There was a tendency towards improved accuracy with greater experience. Ability to predict repetitions to MF is not perfectly accurate among most trainees though may improve with experience. Thus, RIR should be used cautiously in prescription of RT. Trainers and trainees should be aware of this as it may have implications for the attainment of training goals, particularly muscular hypertrophy. 
1 Title: Ability to predict repetitions to momentary failure is not perfectly accurate, though

2 improves with resistance training experience.

3

4 Running title: Predicted vs actual reps to failure

5

6 Original Research Article

7

8 James Steele*1, Andreas Endres ${ }^{2}$, James Fisher ${ }^{1}$, Paulo Gentil ${ }^{3}$, Jürgen Giessing ${ }^{2}$

9

$10{ }^{1}$ School of Sport, Health, and Social Sciences, Southampton Solent University, UK

11 Institute of Sport Science, University of Koblenz-Landau, Germany

$12{ }^{3}$ Faculty of Physical Education and Dance, Federal University of Goias, Brazil

13

14 Corresponding Author:

15 James Steele

16 Southampton Solent University

17 East Park Terrace

18 Southampton

19 UK

20 Tel: +442382016465

21 Email: James.Steele@solent.ac.uk

22

23 Co-Author contact details:

24 Andreas Endres (endr5386@uni-landau.de)

25 James Fisher (James.Fisher@solent.ac.uk)

26 Paulo Gentil (paulogentil@hotmail.com)

27 Jürgen Giessing (giessing@uni-landau.de) 


\section{Abstract}

32 'Repetitions in Reserve' (RIR) scales in resistance training (RT) are used to control effort but assume people accurately predict performance a priori (i.e. the number of possible repetitions to momentary failure $[\mathrm{MF}])$. This study examined the ability of trainees with different experience levels to predict number of repetitions to MF. One hundred and forty one participants underwent a full body RT session involving single sets to MF and were asked to predict the number of repetitions they could complete before reaching MF on each exercise. Participants under predicted the number of repetitions they could perform to MF (Standard error of measurements [95\% confidence intervals] for combined sample ranged between 2.64 [2.36 to 2.99] and 3.38 [3.02 to 3.83]). There was a tendency towards improved accuracy with greater experience. Ability to predict repetitions to MF is not perfectly accurate among most trainees though may improve with experience. Thus, RIR should be used cautiously in prescription of RT. Trainers and trainees should be aware of this as it may have implications for the attainment of training goals, particularly muscular hypertrophy. 


\section{Introduction}

Resistance training (RT) is an exercise mode evidenced to provide a wide range of health

benefits (Steele et al., 2017). As such, understanding prescription of RT to maximise these outcomes is of considerable interest. One variable which may be of primary importance is the intensity of effort employed i.e. whether or not RT is performed to momentary failure (MF; Steele, 2014; Steele, Fisher, Giessing, and Gentil, 2017). A plethora of recent work shows that when effort is matched by having RT performed to MF, manipulations of other RT variables have a lesser impact upon the magnitude of outcomes. These secondary variables include; load (Morton et al., 2016; Schoenfeld, Peterson, Ogborn, Contreras, and Sonmez, 2015; Schoenfeld, Wilson, Lowery, and Krieger, 2016; Fisher, Ironside, and Steele, 2016), repetition duration (Schoenfeld, Ogborn, and Krieger, 2015), and the use of advanced or complicated training methods such as pre-exhaustion (Fisher, Carlson, Steele, and Smith, 2014), breakdown sets (Fisher, Carlson, and Steele, 2015) or occlusion training (Barcelos et al., 2015; Farup, de Paoli, Bjerg, Riis, Ringgard, and Vissing, 2015).

A number of recent reviews have also concluded that training to MF may confer greater adaptations in strength (Fisher, Steele, Smith, and Bruce-Low, 2011), hypertrophy (Fisher,

73 Steele, and Smith, 2013) and possibly cardiorespiratory fitness (Steele, Fisher, McGuff, Bruce74 Low, and Smith et al., 2012) than training not to MF. Conversely, more recent empirical work has shown contrasting results regarding the efficacy of training to MF (Fisher, Blossom, and Steele, 2016; Giessing, Eichmann, Steele, and Fisher, 2016; Giessing, Fisher, Steele, Rothe, 
77 Raubold, and Eichmann, 2016; Izquierdo-Gabarren, González De Txabarri Expósito, Garcia78 pallarés, Sánchez-medina, De Villarreal, and Izquierdo, 2010, Sampson and Groeller, 2016). Proximity to MF has been considered a determinant of the effort employed during RT, and MF 80 (as a set end-point) has been suggested as the only way to objectively match inter- and intraindividual effort due to the variations in number of repetitions possible prior to MF at the same relative loads i.e. \% one repetition maximum (\%1RM; Steele, 2014; Steele, Fisher, Giessing, and Gentil, 2017). In fact, it has been argued that training to MF is the most appropriate way to control the application of a RT stimulus (Dankel et al., 2016).

However, the current body of research has typically considered this variable dichotomously (i.e. people training 'to MF' or 'not to MF'). As a result, the dose-response nature of sub maximal intensities of effort resulting from set end-points occurring at different proximities to MF is unclear. Furthermore, it is unclear whether there is a threshold of relative effort which optimises adaptations. As such, to understand sub maximal effort some have developed scales to assess effort during RT relative to MF (Hackett, Johnson, Halaki, and Chow 2012; Hackett, Cobley, Davies, Michael, and Halaki, 2016; Zourdos et al., 2016; Helms, Cronin, Storey, and Zourdos, 2016). These 'Repetitions in Reserve' (RIR) scales are designed as a way of assessing/controlling relative effort by participants estimating how many repetitions they can perform before reaching MF.

In comparison with traditional rating of perceived effort (RPE) scales, RIR scales appear more likely to offer valid representations of effort when training to, or close to, MF (Helms et al., 2016) whereas traditional RPE often yields far less accurate ratings under such conditions (Hackett et al., 2012). Indeed, even when training to MF, traditional RPE is often less than maximal (Steele, Fisher, McKinnon, and McKinnon, 2017). This in combination with the 
100 considerable inter- and intra-individual variations in number of repetitions possible prior to MF

101 at the same relative loads suggests that RIR scales may offer an improvement in control of effort

102 during RT compared with either use of \%1RM or traditional RPE.

103 However, the use of these scales assumes that trainees are able to accurately predict the 104 number of repetitions they could perform to MF with a particular load. Studies which have 105 shown differences between groups training to MF compared with those not training to MF 106 (where the participants were instructed to stop at the point they predicted MF on the next 107 repetition), might be explained by the participants' inability to accurately predict MF (i.e. they 108 actually stopped $>1$ repetitions away from MF; Giessing, Eichmann, Steele, and Fisher, 2016; 109 Giessing et al., 2016). A study by Hackett et al (2012) supports this, and revealed that trained 110 participants were not perfectly accurate at predicting the number of repetitions they could 111 perform to MF using a RIR scale, although their accuracy improved with subsequent sets. This 112 would question the value of using 'intuitive' approaches to RT such as the RIR scales. Ability to 113 predict proximity to MF may improve with training experience and thus it has been noted that 114 the use of RIR scales may present greater value in experienced trainees (Helms et al., 2016). 115 However, a follow-up study from Hackett et al. (2016) study suggested that previous RT experience did not affect ability to predict proximity to MF.

In light of the potential value of training to MF, as well as the introduction of recent RIR scales to control RT effort, it is of interest to examine trainees with differing levels of RT experience in their ability to predict the number of repetitions they can perform to MF. Further, examination of this in controlled yet ecologically valid conditions such as their usual gym environment offers considerable practical information. As such the aim of this study was to compare predicted with actual repetitions to MF in participants with a range of RT experience. 
2. Methods

2.1. Experimental approach to the Problem

Participants in this study underwent a single RT trial in order to examine whether they

127

128

129

130

131

132

133

134

135

136

137

\section{2. $\quad \underline{\text { Participants }}$}

One hundred and forty-one participants (males $n=72$, age 29 \pm 10 years; females $n=69$, age $25 \pm 8$ years) were recruited from the existing membership pool of a private exercise facility in Germany. Participants were required to have no medical condition for which RT would be contraindicated, and were grouped based upon duration of previous RT experience; $<1.5$ months (orientation, $n=15$ ), 1.5 to 6 months (beginner, $n=21$ ), 6 to 12 months (experienced, $n=21$ ), 12 to 36 months (advanced, $n=42$ ), and $>36$ months (expert, $n=42$ ). Written informed consent was provided by all participants and the study was ethically approved by the author's institution.

\section{3. $\quad$ Procedures}

Participants underwent a single RT session involving the following exercises: seated row, chest press, leg press, elbow flexion, and pulldown, all using selectorised resistance machines, and sit-ups using additional free weight loading. All participants were required to have been performing these exercises in their pre-existing training programs and to have the current 
146 training load they were using recorded in their training logs. Participants performed a single set

147 of each exercise to concentric MF according to recent definitions of this concept i.e. the set

148 ending when the trainee reached the point where, despite attempting to do so, they could not

149 complete the concentric portion of their current repetition without deviation from the prescribed

150 form of the exercise (Steele, Fisher, Giessing, and Gentil, 2017). Participants were informed to

151 use the repetition duration they normally used during training for each exercise, to retain

152 familiarity. Exercises were performed in the order that the participants typically performed them

153 in their current training based upon their recorded training logs and participants were permitted

154 to rest between each exercise for as long they typically would or felt necessary to ensure

155 maximal performance on the subsequent exercise. This was also to ensure that participant's

156 predictions were based upon the RT conditions that they had previously experienced. All

157 exercises were supervised by one of the investigators who observed the participants whilst they

158 performed the exercise without verbal encouragement so as to ensure consistency across

159 participants. The investigator counted repetitions in their head and then noted these without the

160 participant's knowledge. Prior to beginning each exercise participants were asked to consider the

161 current load they were training with and to provide a prediction of the number of repetitions they

162 could complete before reaching MF. Participants were informed that this was defined as the

163 number of repetitions performed with the current load whilst continuing to the point where,

164 despite the greatest effort and attempting to do so, they could not complete the current repetition

165 (i.e. what repetition number they thought they would reach MF on). Participants were also asked 166 to report their current training goals.

167

$168 \quad$ 2.4. $\quad \underline{\text { Statistical Analysis }}$ 

error of measurement (SEM) and 95\% confidence intervals (CI) in order to provide an absolute

171 indication of the agreement between the variables. This was performed for each exercise.

172 Calculations were performed using Microsoft Office Excel 2013 (Microsoft Corporation,

173 Redmond, WA, USA) and spreadsheets for analysis of validity by Hopkins (2015) were used.

174 Actual repetitions were considered the 'criterion' and predicted repetitions where considered the 175 'practical'.

\section{Results}

Descriptive statistics suggested that on average participants under predicted the number of repetitions they could perform to MF (table 1). For the combined sample SEMs $(95 \% \mathrm{CIs})$ were 2.91 (2.61 to 3.30 ) for the chest press, 2.64 (2.36 to 2.99$)$ for the elbow flexion, 3.38 (3.02 to 3.83) for the leg press, 2.95 (2.64 to 3.35) for the pulldown, 2.71 (2.43 to 3.08) for the seated row, and 3.36 (3.00 to 3.80) for the sit-up. SEMs and 95\%CIs are reported in table 2 for each exercise and group. There was a tendency towards improved accuracy in predicting actual repetitions to MF with greater experience across most exercises evidenced by reduced SEMs and narrower ranges between upper and lower 95\%CIs. The training goal of muscular hypertrophy was reported with the highest frequency in the combined participant sample and all groups. Table 3 shows the training goals for each group by frequency.

\section{Discussion}

The current study examined the ability of participants to predict the number of repetitions

they could perform to MF, with a given load, across a number of exercises and range of levels of 
192 experience. It was anticipated that participants would not be perfectly accurate in predicting 193 actual repetitions to MF, in spite of performing exercises and using loads with which they were 194 familiar. It was also hypothesised that there would be increased accuracy with greater RT 195 experience. Descriptive data suggested participants on average under predicted the number of

196

198

200

201

202

203

204

205

206

207

208

209

210

211

212

213

214

repetitions they could perform to $\mathrm{MF}$, though the average difference was reduced with greater experience. The SEMs indicated that participants indeed were not perfectly accurate at predicting repetitions to MF with SEMs for the combined sample ranging from 2.64 to 3.38 repetitions. In contrast with the descriptive data, SEMs suggested this was the case even for groups with greater experience, although there did still appear to be an improvement in accuracy with greater experience across most exercises. Considering the predominant training goal reported by the participants in this study (muscular hypertrophy), a less than perfectly accurate ability to predict repetitions to MF may have implications for achieving this goal.

Training to MF involves giving a maximal effort and is also anecdotally associated with higher discomfort. The less than perfectly accurate predictive ability reported herein may be a result of participants anchoring their prediction based upon discomfort. As we have recently noted in several papers (Steele, 2014; Steele, Fisher, McKinnon, and McKinnon, 2017; Steele, Fisher, Giessing, and Gentil, 2017), and as have others, differentiation between perceptions of effort and discomfort are important (Abbiss, Peiffer, Meeusen, and Skorski, 2015; Marcora, 2009; Smirnaul, 2012) particularly within RT (Steele, 2014; Steele, Fisher, McKinnon, and McKinnon, 2017). In studies using traditional rating of perceived exertion scales higher ratings are given, despite conditions being controlled by supposedly training to MF, with lower loads for lower body exercise (Shimano et al., 2006), as set volume increases (Silva et al., 2014), with increased volume-load (Pritchett, Green, Wickwire, Pritchett and Kovacs et al., 2009), and with 
215 increased work rate (Hiscock, Dawson, Donnelly, and Peeling, 2016; Hiscock, Dawson and

216 Peeling, 2015) supporting that participants may have expressed their feelings of increasing

217 discomfort (Steele, 2014; Steele, Fisher, McKinnon, and McKinnon, 2017; Steele, Fisher,

218 Giessing, and Gentil, 2017).

219 In some studies there have been attempts to differentiate between effort and discomfort

220 during RT. Though participants appear able to report different values for each, there is a similar

221 pattern for both responses. Hollander et al $(2003,2008)$ found that, though effort is typically

222 reported as being higher than discomfort (the authors used the term pain) under a range of RT

223 conditions (different loads and muscle actions), both respond in a similar pattern. Such a

224 relationship may be inherent; however, perception of effort is independent from afferent

225 feedback mechanisms (Marcora, 2009). This would seem to disagree with observations of higher

226 perceived efforts under conditions known anecdotally to induce higher feelings of discomfort

227 (e.g. fatiguing low load lower body exercise). It is possible that participants were either

228 consciously or unconsciously anchoring their effort and discomfort responses upon one another.

229 When instructed to differentiate the two, participants are able to do so during RT (Steele Fisher,

230 McKinnon, and McKinnon, 2017; Fisher, Ironside, and Steele, 2016; Fisher, Farrow, and Steele,

231 2017). But there appears to be a tendency to anchor one upon the other without such instruction.

232 Anchoring of perception of effort upon discomfort thus may have implications for

233 whether a person is truly training to, or close to enough to, MF. Another point to consider is that

234 participants in this study likely based their prediction upon prior experience of training whilst

235 unsupervised as most persons train in this manner. Thus, the not perfectly accurate predictive

236 ability of participants in this study might reflect that under unsupervised conditions participants

237 are not reaching MF during training despite thinking that they may be, possibly due to the 
238 discomfort associated with such training. As such, persons training alone may find difficulty in 239 training to MF unless highly self-motivated. Numerous studies report that strength and body 240 composition changes are poorer when participants train unsupervised versus training under 241 supervision (Coutts, Murphy, and Dascombe, 2004; Gentil and Bottaro, 2010; Mazzetti et al., 242 2000). When participants self-select RT load they often choose to train with lower loads than 243 those recommended (Elsangedy, Krause, Krinski, Alves, Hsin Nery Chao, and da Silva, 2013; 244 Glass and Stanton, 2004) and, considering the typical ranges of repetitions performed to MF by 245 trainees at these loads (Shimano et al., 2006), are likely not training anywhere close to MF. 246 Indeed, the RPE reported when participant's self-select load and repetition range, in addition to 247 trainer observation, support this (Glass and Stanton, 2004). Instead, under supervision 248 participants are more likely to train with heavier loads but also to report higher RPE (Ratamess, 249 Faigenbaum, Hoffman, and Kang, 2008). In fact it has been suggested that the poorer adaptations 250 as a result of unsupervised training may be due to participants not training with sufficient 251 proximity to MF and thus with lower effort (Gentil and Bottaro, 2010).

Evidently there may be implications for whether a person is able to achieve their training 253 goals if they are unable to accurately perceive whether they are training to true MF or not. However, as noted there is disagreement within the literature as to whether performing RT to MF is indeed desirable and further that the consideration of MF in a dichotomous fashion (i.e. people training 'to MF' or 'not to MF') renders difficulty in understanding the nature of sub-maximal 257 efforts during RT (Steele, Fisher, Giessing and Gentil, 2017). As a result, RIR scales have been 258 developed to be used in controlling sub maximal effort in RT as an improvement upon the 259 typical \%1RM and traditional RPE based approaches (Hackett et al., 2012, 2016; Zourdos et al., 260 2016). The results reported here are in agreement with other research (Hackett et al., 2012, 2016) 
261 that participants are likely not perfectly accurate at predicting the number of repetitions they can

262 perform to MF and thus suggest there may be reason to question the value of RIR scales. The use

263 of RIR scales assumes a trainee is able to accurately predict the number of repetitions they could

264 perform to MF. However, if a trainee is not perfectly accurate at making such a prediction then it

265 is likely that they will be systematically training with a lower than desired effort level which may

266 impact upon their adaptation to RT.

267 For untrained persons this may not be of considerable practical concern. In this case, even

268 when using a lower than intended effort during RT on an individual set, cumulative fatigue can

269 be induced by increased volume resulting in an increased effort, and thus closer proximity to

270 MF, in later sets (Fisher, Blossom and Steele, 2016; Giessing, Fisher, Steele, Rothe et al., 2016).

271 However, experience may a play role in a trainee's ability to predict proximity to MF and

272 indeed the results reported here support this notion. There was a relationship between the level of

273 experience of participants and the SEMs and width of 95\%CIs found, with the most experienced

274 group under predicting by $\sim 1-2$ repetitions compared with the least experienced under predicting

275 by $\sim 4-5$ repetitions. Hackett et al (2012) found experienced trainees ( $8 \pm 3$ years RT experience)

276 were initially not perfectly accurate at predicting repetitions to MF using the RIR scale over the

277 first 1-2 sets (mean difference ranging 0.8 to 1.9 repetitions). However, on average accuracy

278 improved in later sets. This suggests that, similar to our findings, even experienced trainees are

279 still not perfectly accurate at predicting repetitions to MF, yet acute practice/experience appears

280 to improve predictive ability. Further supporting the effect of experience, Zourdos et al (2016)

281 found that their RIR scale reflected more experienced lifters giving a more accurate estimation of

282 their effort, particularly when using heavier loads, based upon average repetition velocities. Thus 
283 the novice trainees in their study likely overestimated their effort and therefore were likely under

284 predicting how many repetitions away from MF they were.

Though increased experience would appear to increase predictive ability experienced trainees still under estimate by $\sim 1-2$ repetitions. Thus using sub-maximal effort based RT prescriptions based upon RIR scales will result in most training at a lower than intended effort. For trained persons this may have a bigger impact upon adaptations. When attempting to stop a set of repetitions at a set end-point corresponding to a 'self determined repetition maximum' (where the participants were instructed to stop at the point they predicted MF on the next repetition) strength and hypertrophic outcomes may be sub-optimal (Giessing, Eichmann, Steele, and Fisher, 2016). Thus, the use of 'intuitive' approaches that involve a person's ability to accurately predict the number of repetitions they could perform to MF may be questionable as an approach to prescribing and controlling effort in RT. However, the use of self determined repetition maximum based training compared with training to MF in experienced participants has only been examined with use of single set approaches (Giessing, Fisher, Steele, Rothe et al., 2016). As accuracy of predictive ability improves with multiple sets of an exercise (Hackett et al., 2012) then RIR scales may have more utility in multiple set RT programs. Indeed, similarly to in untrained populations (Fisher, Blossom and Steele, 2016; Giessing, Fisher, Steele, Rothe et al., 2016), even if training with a systematically lower than intended effort, the use of multiple sets, and thus the accumulation of fatigue, combined with improved predictive ability, makes it likely that in later sets trainees would be closer to achieving desired intensities of effort. As such, though even 'expert' participants in this study under predicted by $\sim 1-2$ repetitions, it is likely that this represents an acceptable degree of error if RIR scale based approaches to training are being 305 utilised in multiple set routines. However, it seems as though even this degree of error has 
306 implications when using single set routines and as such predictive ability appears unacceptable

307 for this approach.

308 It is worth considering the strengths and limitations of the present study. Firstly the

309 present study was able to recruit a large sample size sufficient for examining validity/agreement

310 between different measures (Hopkins, 2000). Due to this we were also able to sub group into a

311 range of different experience levels. However, in order to achieve this large sample, participants

312 were recruited from a private facility and testing conducted at this facility. This meant

313 participants performed the testing using their current training equipment and load and, based

314 upon the average repetitions, relative loads typically increased with experience. As such, the

315 effects of experience level on the SEMs reported may be confounded as a result of differing

316 ability to predict repetitions to MF when training using heavier or lighter loads. Greater

317 predictive ability may therefore occur with heavier loads (Zourdos et al., 2016; Helms, Brown,

318 Cross, Storey, Cronin and Zourdos, 2017). This may be reflective of the conflation between

319 effort and discomfort described above as greater perceived discomfort occurs with lower load RT

320 (Fisher, Ironside et al., 2016; Fisher, Farrow, and Steele, 2017). Future research should examine

321 the impact that manipulation of other RT variables such as load, and its interaction with

322 perceived discomfort, has upon ability to accurately predict repetitions to MF. A final limitation

323 could be that we asked participants to predict the number of repetitions they could perform to

324 MF prior to the execution of the exercise. Prior studies have asked participants during the

325 execution of the set (Hackett et al., 2012; 2016) and thus participants may be able to make better

326 predictions during the gestalt experience of actually performing the exercise. However, it should

327 be noted that in studies where participants have attempted to stop one repetition prior to MF sub

328 optimal adaptations have still been reported (Giessing, Eichmann, Steele, and Fisher, 2016; 
329 Giessing, Fisher, Steele, Rothe, Raubold, and Eichmann, 2016). Also, we did not control when

330 the participant's penultimate training session prior to the testing sessions were, nor did we

331 control and match other factors such as time of day, diet, sleep, etc. As such, there is still scope

332 for further work to identify what factors may positively or negatively impact upon a person's

333 predictive ability in performing repetitions to MF.

334

335

336

337

338

339

340

341

342

343

344

345

346

347

348

349

350

351

\section{Conclusion}

Management of effort within RT by manipulation of whether a trainee reaches MF or not is a common approach by trainees and practitioners. Effort, and thus proximity to MF, may have implications for the optimisation of adaptations, in particular hypertrophy which for most commercial gym attendees is the most common training goal. Recently, RIR scales have been promoted as a means of controlling this and represent an improvement on \%1RM and traditional RPE scales. However, they assume the trainee can make accurate predictions regarding their ability to perform repetitions to MF. The findings of the present study reveal that ability to predict repetitions to MF is not perfectly accurate amongst most trainees. However, there may be some increase in predictive ability with greater RT experience.

These results have implications regarding training adaptations from RT as most persons train unsupervised and thus are likely not training to actual MF in their current training programs. Further, for those not employing MF in their training but instead using sub-maximal efforts based upon proximity to MF, it is likely that they are systematically training with a lower than intended effort. These results suggest that RIR scales should be used with caution in most trainees. It appears that experience may improve a trainee's ability to predict repetitions to MF and therefore RIR scales may be more appropriate for experienced trainees. Lastly, these results 
352 apply to single set applications of RT. Prior research suggests with multiple sets predictive

353 ability increases. As such, RIR scales may have the greatest utility in experienced trainees using 354 multiple set RT programs.

355 
357

358

359

360

361

362

363

364

365

366

367

368

369

370

371

372

373

374

375

376

377

378

\section{$\underline{\text { References }}$}

1. Abbiss, C. R., Peiffer, J. J., Meeusen, R., \& Skorski, S. (2015). Role of ratings of perceived exertion during self-paced exercise: What are we actually measuring? Sports Medicine, 45, 1235-1243. doi: 10.1007/s40279-015-0344-5

2. Barcelos, L. C., Nunes, P. R., de Souza, L. R., de Oliviera, A. A., Furlanetto, R., Marocolo, M., \& Orsatti, F. L. (2015). Low-load resistance training promotes muscular adaptation regardless of vascular occlusion, load, or volume. European Journal of Applied Physiology, 115, 1559-1568. doi: 10.1007/s00421-015-3141-9

3. Coutts, A. J., Murphy, A. J., \& Dascombe, B. J. (2004). Effect of direct supervision of a strength coach on measures of muscular strength and power in young rugby league players. Journal of Strength and Conditioning Research, 18, 316-323.

4. Dankel, S. J., Jessee, M. B., Mattocks, K. T., Mouser, J. G., Counts, B. R., Buckner, S. L., \& Loenneke, J. P. (2016). Training to fatigue: The answer for standardization when assessing muscle hypertrophy? Sports Medicine, Epub ahead of print.

5. Elsangedy, H. M., Krause, M. P., Krinski, K., Alves, R. C., Hsin Nery Chao, C., \& da Silva, S. G. (2013). Is the self-selected resistance exercise intensity by older women consistent with the American College of Sports Medicine guidelines to improve muscular fitness? Journal of Strength and Conditioning Research, 27, 1877-84. doi: 10.1519/JSC.0b013e3182736cfa

6. Farup, J., de Paoli, F., Bjerg, K., Riis, S., Ringgard, S., \& Vissing, K. (2015). Blood flow restricted and traditional resistance training performed to fatigue produce equal muscle 
hypertrophy. Scandinavian Journal of Medicine and Science in Sports, 25, 754-63. doi:

7. Fisher, J. P., Blossom, D., \& Steele, J. (2016). A comparison of volume equated knee extensions to failure, or not to failure, upon rating of perceived exertion and strength adaptations. Applied, Physiology, Nutrition, and Metabolism, 41, 168-174. doi: 10.1139/apnm-2015-0421

8. Fisher, J. P., Carlson, L., \& Steele, J. (2015). The effects of breakdown set resistance training on muscular performance and body composition in young males and females. Journal of Strength and Conditioning Research, 30, 1425-1432. doi: 10.1519/JSC.0000000000001222

9. Fisher, J. P., Carlson, L., Steele, J., \& Smith, D. (2014). The effects of pre-exhaustion, exercise order, and rest intervals in a full-body resistance training intervention. Applied, Physiology, Nutrition, and Metabolism, 39, 1-6. doi: 10.1139/apnm-2014-0162

10. Fisher, J., Farrow, J., \& Steele, J. (2017). Acute fatigue, and perceptual responses to resistance exercise. Muscle and Nerve, Epub ahead of print. doi: 10.1002/mus.25645

11. Fisher, J., Ironside, M., \& Steele, J. (2016). Heavier- and lighter- load resistance training to momentary failure produce similar increases in strength with differing degrees of discomfort. Muscle and Nerve, Epub ahead of print. doi: 10.1002/mus.25537

12. Fisher, J., Steele, J., \& Smith, D. (2013). Evidence-based resistance training recommendations for muscular hypertrophy. Medicina Sportiva, 17, 217-235. doi: $10.5604 / 17342260.1081302$ 
400

401

402

403

404

405

406

407

408

409

410

411

412

413

414

415

416

417

418

419

420

13. Fisher, J., Steele, J., Smith, J., \& Bruce-Low, S. (2011). Evidence based resistance training recommendations. Medicina Sportiva, 15, 147-162. doi: 10.2478/v10036-0110025-x

14. Gentil, P., \& Bottaro, M. (2010). Influence of supervision ratio on muscle adaptations to resistance training in nontrained subjects. Journal of Strength and Conditioning Research, 24, 639-643. doi: 10.1519/JSC.0b013e3181ad3373

15. Giessing, J., Eichmann, B., Steele, J., \& Fisher, J. (2016). A comparison of low volume 'high-intensity-training' and high volume traditional resistance training methods on muscular performance, body composition, and subjective assessments of training. Biology of Sport, 33, 241-249. doi: 10.5604/20831862.1201813

16. Giessing, J., Fisher, J., Steele, J., Rothe, F., Raubold, K., \& Eichmann, B. (2016). The effects of low volume resistance training with and without advanced techniques in trained participants. Journal of Sports Medicine and Physical Fitness, 56, 249-258.

17. Glass, S. C., \& Stanton, D. R. (2004). Self-selected resistance training intensity in novice weightlifters. Journal of Strength and Conditioning Research, 18, 324-327.

18. Hackett, D. A., Cobley, S., Favies, T., Michael, S., \& Halaki, M. (2016). Accuracy in estimating repetitions to failure during resistance exercise. Journal of Strength and Conditioning Research, Epub ahead of print

19. Hackett, D. A., Johnson, N. A., Halaki, M., \& Chow, C. (2012). A novel scale to assess resistance-exercise effort. Journal of Sports Sciences, 30, 1405-1413. doi: $10.1080 / 02640414.2012 .710757$ 
421

422

423

424

425

426

427

428

429

430

431

432

433

434

435

436

437

438

439

440

441

442

443

20. Helms, E. R., Cronin, J., Storey, A., \& Zourdos, M. (2016). Application of the repetitions in reserve-based rating of perceived exertion scale for resistance training. Strength and Conditioning Journal, 38, 42-49

21. Helms, E. R., Brown, S. R., Cross, M. R., Storey, A., Cronin, J., \& Zourdos, M. (2017). Self-rated accuracy of rating of perceived exertion-based load prescription in powerlifters. Journal of Strength and Conditioning Research, Epub ahead of print. doi: 10.1519/JSC. 0000000000002097

22. Hiscock, D. J., Dawson, B., \& Peeling, P. (2015). Perceived exertion responses to changing resistance training programming variables. Journal of Strength and Conditioning Research, 29, 1564-1569. doi: 10.1519/JSC.0000000000000775

23. Hiscock, D. J., Dawson, B., Donnelly, C. J., \& Peeling, P. (2016). Muscle activation, blood lactate, and perceived exertion responses to changing resistance training programming variables. European Journal of Sport Science, 16, 536-544. doi: $10.1080 / 17461391.2015 .1071880$

24. Hollander, D. B., Duran, R. J., Trynicki, J. L., Larock, D., Castracane, V. D., Hebert, E. P., \& Kraemer, R. R. (2003). RPE, pain, and physiological adjustment to concentric and eccentric contractions. Medicine and Science in Sports and Exercise, 35, 1017-1025

25. Hollander, D. B., Kilpatrick, M. W., Ramadan, Z. G., Reeves, G. V., Francois, M., Blakeney, A., Castracane, V. D., \& Kraemer, R. R. (2008). Load rather than contraction type influences rate of perceived exertion and pain. Journal of Strength and Conditioning Research, 22, 1184-1193. doi: 10.1519/JSC.0b013e31816a8bc2

26. Hopkins, W. G. (2000). Measures of Reliability in Sports Medicine and Science. Sports Medicine, 30, 1-15. 
444

445

446

447

448

449

450

451

452

453

454

455

456

457

458

459

460

461

462

463

464

465

27. Hopkins, W. G. (2015). Spreadsheets for analysis of validity and reliability. Sportscience, 19, 36-42 Retrieved from http://sportsci.org/2015/ValidRely.htm. Accessed $12^{\text {th }}$ May 2016

28. Izquierdo-Gabarren, M., Gonzalez De Txbarri Exposito, R., Garcia-pallares, J., SanchezMedina, J., De Villarreal, E. S., \& Izquierdo, M. (2010). Concurrent endurance and strength training not to failure optimises performance gains. Medicine and Science in Sports and Exercise, 42, 1191 - 1199. doi: 10.1249/MSS.0b013e3181c67eec

29. Marcora, S. (2009). Perception of effort during exercise is independent of afferent feedback from skeletal muscles, heart, and lungs. Journal of Applied Physiology, 106, 2060-2062. doi: 10.1152/japplphysiol.90378.2008

30. Mazzetti, S. A., Kraemer, W. J., Volek, J. S., Dunca, N. D., Ratamess, N. A., Gomez, A. L., ... Fleck, S. J. (2000). The influence of direct supervision of resistance training on strength performance. Medicine and Science in Sports and Exercise, 32, 1175-1184.

31. Morton, R. W., Oikawa, S. Y., Wavell, C. G., Mazara, N., McGlory, C., Quadrilatero, J., ... Phillips, S. M. (2016). Neither load nor systemic hormones determine resistance training-mediated hypertrophy or strength gains in resistance-trained young men. Journal of Applied Physiology, 121, 129-138. doi: 10.1152/japplphysiol.00154.2016

32. Pritchett, R. C., Green, J. M., Wickwire, P. J., Pritchett, K. L., \& Kovacs, M. S. (2009). Acute and session RPE responses during resistance training: Bouts to failure at $60 \%$ and 90\% of 1RM. South African Journal of Sports Medicine, 21. 23-26

33. Ratamess, N. A., Faigenabum, A. D., Hoffman, J. R., \& Kang, J. (2008). Self-selected resistance training intensity in healthy women: The influence of a personal trainer. 
466

467

468

469

470

471

472

473

474

475

476

477

478

479

480

481

482

483

484

485

486

487

488

Journal of Strength and Conditioning Research, 22, 103-111. doi: 10.1519/JSC.0b013e31815f29cc

34. Sampson, J. A., \& Groeller, H. (2016). Is repetition failure critical for the development of muscle hypertrophy and strength? Scandinavian Journal of Medicine and Science in Sports, 26, 375-383. doi: 10.1111/sms.12445

35. Schoenfeld, B. J., Ogborn, D. I., \& Krieger, J. W. (2015). Effect of repetition duration during resistance training on muscle hypertrophy: a systematic review and meta-analysis. Sports Medicine, 45, 577-585. doi: 10.1007/s40279-015-0304-0

36. Schoenfeld, B. J., Peterson, M. D., Ogborn, D., Contreras, B., \& Sonmez, G. T. (2015). Effects of low- versus high-load resistance training on muscle strength and hypertrophy in well-trained men. Journal of Strength and Conditioning Research, 29, 2954-2963. doi: 10.1519/JSC.0000000000000958

37. Schoenfeld, B. J., Wilson, J. M., Lowery, R. P., \& Krieger, J. W. (2016). Muscular adaptations in low-versus high-load resistance training: A meta-analysis. European Journal of Sport Science, 16, 1-10. doi:10.1080/17461391.2014.989922.

38. Shimano, T., Kraemer, W. J., Spiering, B. A., Volek, J. S., Hatfield, D. L., Silvestre, R., ... Häkkinen, K. (2006). Relationship between the number of repetitions and selected percentages of one repetition maximum in free weight exercises in trained and untrained men. Journal of Strength and Conditioning Research, 20 819-823

39. Silva, V. L., Azevedo, A. P., Cordeiro, J. P., Duncan, M. J., Cholewa, J. M., SiqueiraFilho, M. A., ... Guimaraes-Ferreira, L. (2014). Effects of exercise intensity on perceived exertion during multiple sets of bench press to volitional failure. Journal of Trainology, 3, 41-46. doi:org/10.17338/trainology.3.2_41 
489

490

491

492

493

494

495

496

497

498

499

500

501

502

503

504

505

506

507

508

509

510

511

40. Smirnaul, B. D. P. C. (2012). Sense of effort and other unpleasant sensations during exercise: clarifying concepts and mechanisms. British Journal of Sports Medicine, 46, 308-311. doi: 10.1136/bjsm.2010.071407

41. Steele, J. (2014). Intensity; in-ten-si-ty; noun. 1. Often used ambiguously within resistance training. 2. Is it time to drop the term altogether? British Journal of Sports Medicine, 48, 586-1588. doi: 10.1136/bjsports-2012-092127

42. Steele, J., Fisher, J., Giessing, J., \& Gentil, P. (2017). Clarity in reporting terminology and definitions of set end points in resistance training. Muscle and Nerve, Epub ahead of print. doi: $10.1002 /$ mus. 25557

43. Steele, J., Fisher, J., McGuff, D., Bruce-Low, S., \& Smith, D. (2012). Resistance training to momentary muscular failure improves cardiovascular fitness in humans: a review of acute physiological responses and chronic physiological adaptations. Journal of Exercise Physiology, 15, 53-80

44. Steele, J., Fisher, J., McKinnon, S., \& McKinnon, P. (2017). Differentiation between perceived effort and discomfort during resistance training in older adults: reliability of trainee ratings of effort and discomfort, and reliability and validity of trainer ratings of trainee effort. Journal of Trainology, 6, 1-8.

45. Steele, J., Fisher, J., Skivington, M., Dunn, C., Arnold, J., Tew, G., ... Winett, R. (2017). A higher effort-based paradigm in physical activity and exercise for public health: making the case for a greater emphasis on resistance training. BMC Public Health, 17, 300

46. Zourdos, M. C., Klemp, A., Dolan, C., Quiles, J. M., Schau, K. A., Jo, E., Helms, E., Esgro, B., Duncan, S., \& Garcia-Merino Blanco, R. (2016). Novel resistance training- 
512

513

514

515

516

517

518

519

520

521

522

523

524

525

526

527

528

529

530

531

532

533

534 specific rating of perceived exertion scale measuring repetitions in reserve. Journal of Strength and Conditioning Research, 30, 267-275. doi: 10.1519/JSC.0000000000001049 
536 
Table $\mathbf{1}$ (on next page)

Descriptive data (mean \pm SD) for each exercise and group 
1 Table 1. Descriptive data (mean $\pm \mathrm{SD}$ ) for each exercise and group

\begin{tabular}{|c|c|c|c|c|c|c|}
\hline & Combined & Orientation & Beginner & Experienced & Advanced & Expert \\
\hline \multicolumn{7}{|l|}{ Chest Press } \\
\hline Predicted & $12.38 \pm 3.45$ & $15.40 \pm 2.77$ & $14.86 \pm 3.00$ & $14.00 \pm 3.46$ & $11.114 \pm 2.55$ & $10.48 \pm 2.86$ \\
\hline Actual & $14.21 \pm 5.52$ & $20.47 \pm 6.36$ & $18.76 \pm 6.78$ & $15.86 \pm 4.16$ & $12.17 \pm 3.39$ & $10.93 \pm 3.17$ \\
\hline \multicolumn{7}{|l|}{ Elbow Flexion } \\
\hline Predicted & $11.53 \pm 2.99$ & $15.40 \pm 2.77$ & $13.38 \pm 2.85$ & $12.67 \pm 3.07$ & $10.60 \pm 2.06$ & $9.60 \pm 1.58$ \\
\hline Actual & $12.48 \pm 4.32$ & $18.20 \pm 4.95$ & $16.57 \pm 4.65$ & $13.14 \pm 2.67$ & $10.74 \pm 2.36$ & $9.81 \pm 2.11$ \\
\hline \multicolumn{7}{|l|}{ Leg Press } \\
\hline Predicted & $12.50 \pm 3.41$ & $15.40 \pm 2.77$ & $14.95 \pm 3.14$ & $13.67 \pm 3.65$ & $11.45 \pm 2.62$ & $10.71 \pm 2.79$ \\
\hline Actual & $16.40 \pm 6.29$ & $23.87 \pm 7.04$ & $22.19 \pm 5.75$ & $17.62 \pm 4.50$ & $14.52 \pm 4.13$ & $12.10 \pm 3.79$ \\
\hline \multicolumn{7}{|l|}{ Pulldown } \\
\hline Predicted & $12.48 \pm 3.47$ & $15.40 \pm 2.777$ & $15.27 \pm 3.13$ & $13.29 \pm 3.36$ & $11.55 \pm 2.93$ & $10.57 \pm 2.77$ \\
\hline Actual & $13.81 \pm 5.19$ & $20.27 \pm 6.92$ & $17.86 \pm 5.76$ & $14.57 \pm 4.04$ & $11.95 \pm 2.81$ & $10.95 \pm 2.83$ \\
\hline \multicolumn{7}{|l|}{ Seated Row } \\
\hline Predicted & $12.38 \pm 3.45$ & $15.40 \pm 2.77$ & $14.86 \pm 3.00$ & $14.00 \pm 3.46$ & $11.14 \pm 2.55$ & $10.48 \pm 2.86$ \\
\hline Actual & $14.09 \pm 5.29$ & $19.67 \pm 5.38$ & $18.33 \pm 5.80$ & $15.81 \pm 4.24$ & $12.26 \pm 3.60$ & $10.93 \pm 3.48$ \\
\hline \multicolumn{7}{|l|}{ Sit-up } \\
\hline Predicted & $14.62 \pm 3.05$ & $15.93 \pm 2.40$ & $16.43 \pm 2.62$ & $15.71 \pm 2.99$ & $13.88 \pm 2.92$ & $13.43 \pm 3.16$ \\
\hline Actual & $16.99 \pm 3.65$ & $18.73 \pm 5.19$ & $17.71 \pm 3.99$ & $17.76 \pm 2.02$ & $17.31 \pm 3.23$ & $15.29 \pm 3.39$ \\
\hline
\end{tabular}

2 
Table 2 (on next page)

SEMs and $95 \%$ Cls for each exercise and group. 
1 Table 2. SEMs and 95\%CIs for each exercise and group.

\begin{tabular}{lccccc}
\hline & Orientation & Beginner & Experienced & Advanced & Expert \\
\hline Chest Press & $4.33(3.14$ to & $4.00(3.04$ to & $2.58(1.96$ to & $1.91(1.57$ to & $1.57(1.29$ to \\
& $6.97)$ & $5.84)$ & $3.76)$ & $2.45)$ & $2.00)$ \\
Elbow Flexion & $4.30(3.12$ to & $6.39(4.86$ to & $1.51(1.15$ to & $1.95(1.60$ to & $1.27(1.04$ to \\
& $6.93)$ & $9.33)$ & $2.20)$ & $2.50)$ & $1.62)$ \\
Leg Press & $4.98(3.61$ to & $3.37(2.56$ to & $3.07(2.34$ to & $2.49(2.05$ to & $1.73(1.42$ to \\
& $8.03)$ & $4.92)$ & $4.49)$ & $3.19)$ & $2.21)$ \\
Pulldown & $4.15(3.01$ to & $3.89(2.96$ to & $2.49(1.89$ to & $1.83(1.50$ to & $1.35(1.11$ to \\
Seated Row & $6.68)$ & $5.68)$ & $3.64)$ & $2.34)$ & $1.73)$ \\
& $4.51(3.27$ to & $3.50(2.66$ to & $2.28(1.73$ to & $2.06(1.69$ to & $1.71(1.40$ to \\
Sit-up & $7.27)$ & $5.11)$ & $3.33)$ & $2.63)$ & $2.19)$ \\
\hline & $5.13(3.72$ to & $4.08(3.10$ to & $2.06(1.57$ to & $2.87(2.36$ to & $2.29(1.88$ to \\
& $8.26)$ & $5.96)$ & $3.01)$ & $3.68)$ & $2.94)$ \\
\hline
\end{tabular}

3

4

5

6

7 
Table $\mathbf{3}$ (on next page)

Participants training goals by frequency. 
1 Table 3. Participants training goals by frequency.

\begin{tabular}{lcccccc}
\hline & Combined & Orientation & Beginner & Experienced & Advanced & Expert \\
& $(n=141)$ & $(n=15)$ & $(n=21)$ & $(n=21)$ & $(n=42)$ & $(n=42)$ \\
\hline Fitness & $6(4.3 \%)$ & $1(6.6 \%)$ & $2(9.5 \%)$ & $2(9.5 \%)$ & 0 & $1(2.4 \%)$ \\
Maintenance & $3(2.1 \%)$ & 0 & $1(4.8 \%)$ & 0 & $1(2.4 \%)$ & $1(2.4 \%)$ \\
Muscular definition & $8(5.7 \%)$ & 0 & $1(4.8 \%)$ & $1(4.8 \%)$ & $2(4.8 \%)$ & $3(7.1 \%)$ \\
Muscular & $107(75.9 \%)$ & $10(66.6 \%)$ & $14(66.6 \%)$ & $14(66.6 \%)$ & $36(85.7 \%)$ & $33(78.6 \%)$ \\
hypertrophy & & & & & & \\
Strength & $4(2.8 \%)$ & 0 & $1(4.8 \%)$ & 0 & $1(2.4 \%)$ & $2(4.8 \%)$ \\
Weight loss & $13(9.2 \%)$ & $4(26.6 \%)$ & $2(9.5 \%)$ & $4(19.0 \%)$ & $1(2.4 \%)$ & $2(4.8 \%)$ \\
\hline
\end{tabular}

2 